\title{
Complementary Split-Ring Resonator with Improved Dielectric Spatial Resolution
}

\author{
Gertjan Maenhout, Tomislav Markovic, Ilja Ocket, and Bart Nauwelaers
}

\begin{abstract}
A nondestructive complementary split ring resonator (CSRR), working in the 0.5 to $6 \mathrm{GHz}$ frequency range, is presented in this work. Three CSRR designs, on the same $5 \mathrm{~mm}$ by $5 \mathrm{~mm}$ footprint, with different internal dimensions were designed, fabricated, and measured. The results demonstrate a large shift in resonant frequency, up to $2.544 \mathrm{GHz}$, over a broad relative permittivity range from 1 to 80 , and a large penetration depth, up to $7 \mathrm{~mm}$, into the material under test. Secondly, this work presents a deconvolutionbased method to increase the spatial resolution of the pre-

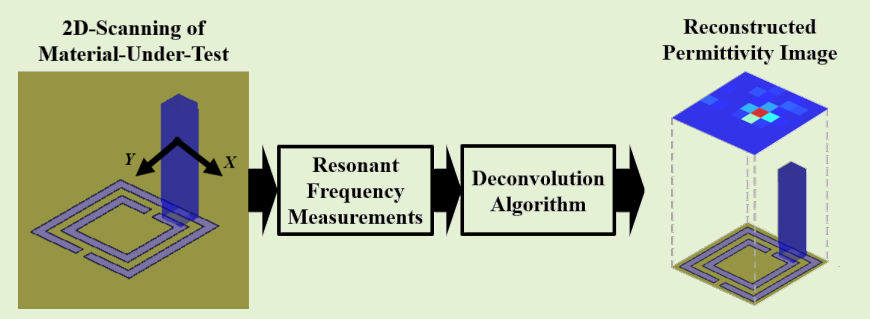
sented sensors compared to other sensing structures and algorithms. A conformal mapping function relating the resonant frequency to the relative permittivity was fitted through measurements and the point spread function (PSF) was derived from a known dielectric permittivity distribution. Experimental results demonstrated that the extracted PSF was able to reconstruct the dielectric distribution in six different scenarios. Additionally, a spatial resolution of 2 mm has been established according to the Rayleigh criterion, and objects smaller than the size of the sensor have been reconstructed as well. Objects with a different permittivity values can be distinguished as well. The reconstructed images of the objects have a good signal-to-clutter ratio up to $12.0 \%$, which results from a smooth recovered background, and no spatial offset was present between the original and reconstructed objects.
\end{abstract}

Index Terms-Deconvolution, dielectric measurement, image reconstruction, microwave sensors, nondestructive testing, spatial resolution.

\section{INTRODUCTION}

C OLORECTAL cancer (CRC) is currently the cancer with the second-highest prevalence worldwide, accounting for more than 1.85 million new cases and causing more than 880000 deaths in 2018 [1], [2]. These numbers are expected to rise even further due to the adaptation of a more Western lifestyle in current low- and middle-income countries [3]. Therefore, there is a high need for colorectal cancer screening tests that are aiming to reduce mortality by increasing the detection of cancer in early stages [4]. An integral part in screening strategies is a colonoscopy, which is the golden standard to detect polyps, and colonoscopies have shown to reduce the incidence of colorectal cancer resulting in a reduced mortality [5], [6]. Nevertheless, during colonoscopic

Manuscript received Month xx, 2020; revised Month xx, 2020; accepted Month Xx, 2020. Date of publication Month Xx, 2020; date of current version Month Xx, 2020. This work is supported in part by the Research Foundation Flanders (FWO) SB PhD fellowship under grant number $1 \mathrm{~S} 23918 \mathrm{~N}$ (G. Maenhout). The associate editor coordinating the review of this article and approving it for publication was Prof. TBD. (Corresponding author: G. Maenhout)

G. Maenhout, T. Markovic, and B. Nauwelaers are with the Department of Electrical Engineering (ESAT), KU Leuven, Kasteelpark Arenberg 10, Box 2444, 3001, Leuven, Belgium (e-mail: gertjan.maenhout@esat.kuleuven.be; tomislav.markovic@esat.kuleuven.be; bart.nauwelaers@esat.kuleuven.be).

T. Markovic and I. Ocket are with imec, Kapeldreef 75, 3001 Heverlee, Belgium (e-mail: tomislav.markovic@imec.be; ilja.ocket@imec.be).

Digital Object Identifier TBD examinations, polyps are missed at a rate varying from $6 \%$ to $27 \%$ [7]. Furthermore, regarding flat adenoma's (Paris classification [8]), the miss rate can reach up to $44.3 \%$ [9]. This high miss rate has led to the development of new devices and techniques, mostly related to the optical part of the system, to improve colon polyp and adenoma detection [10].

Lately, microwave- and millimeter-wave-based dielectric spectroscopy has increasingly been employed in cancer research [11], [12]. These electromagnetic (EM) waves are directed towards a material-under-test (MUT), e.g. a tissue sample or a body part, and will be scattered depending on the differences in dielectrical properties of the MUT. This scattering can be collected by antennas and sensors, and used to reconstruct the distribution of the dielectric properties in the MUT. Theoretically, the aforementioned electromagnetic scattering is likewise influenced by the permeability of the MUT. However, since no contrast in permeability is present in biological tissue, this will not be further discussed. One of the observations throughout several dielectric measurements is the statistically significantly higher permittivity value of malignant tissues compared to the permittivity value of healthy tissues [13]. This effect can be potentially assigned to the greater extra- and intra-cellular water content in tumors, and the more dividing nature of malignant cells. Given the clear advantages of low cost, non-invasive operation, and the ability to penetrate deeper into the tissue than visible light, dielectric spectroscopy can become a worthy clinical complement to nowadays exist- 
ing techniques. Therefore, these techniques have been widely applied to breast cancer, the most common cancer in today's society [1]. A substantial contrast in dielectric properties has been established between normal and malignant breast tissue over a broad frequency range from 0.5 to $50 \mathrm{GHz}$ [14]. Based on this dielectric contrast, several microwave imaging systems [15] and algorithms [16] for breast cancer detection have been developed which resulted in multiple clinical prototypes [17].

In 2014, L. Zhang et al. reported the discrimination of several colorectal cell lines based on their cancer cell stage, i.e. agressiveness, using microwave biosensors [18]. A clear correlation between the dielectric permittivity of a cell and its aggressiveness was established, i.e. the higher the measured permittivity of the cell, the more aggressive it was. From 2016 to 2018, three different measurements campaigns were conducted to measure the dielectric properties of healthy colorectal tissue, colon polyps, and malignant colorectal tissue samples from surgery resections [19]-[21]. All three campaigns concluded that there exists a statistically significant difference in permittivity between healthy colorectal tissue, colon polyps, and malignant colorectal tissue and that the difference is consistent for frequencies from $50 \mathrm{MHz}$ to $20 \mathrm{GHz}$, which was expected from the general theory as well as from the prior experiments with colorectal cell lines. Additionally, we can conclude that dielectric losses increase as well with increasing cancer cell stages [19]-[21].

A first system towards microwave imaging based on dielectric tissue properties assisting in colon polyp detection proposes to attach an antenna applicator to the tip of a conventional endoscope [22]. In our group, we envision the use of a balloon with deposited metal layers that, when inflated, contacts the colorectal wall. These inflatable balloons are used regularly during colonoscopic examination and they have been proven to be biocampatible and suitable for RF signals for cardiac electrophysiological mapping and ablation therapy [23]. Additionally, the use of microwave sensors is interesting due to its dual-purpose opportunities: using microwave spectroscopy to investigate the nature of the sample as well as performing microwave hyperthermia to apply elevated temperatures for a sufficiently long period of time [24] or microwave ablation to induce cellular death via coagulation necrosis [25].

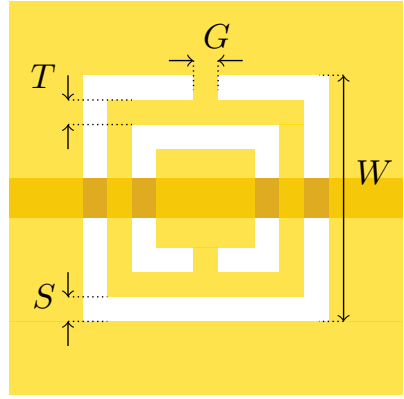

(a)
This work focuses on the design of a sensor for microwave detection of colorectal polyps, which could be later implemented on an inflatable balloon. Throughout this work, the inflatable balloon is considered as a thin substrate with potential for depositing metal on both sides without the option to include vias and is represented as a rigid substrate. Additionally, we examine the microwave imaging capabilities of the proposed sensor. In Section II, we elaborate on the selected sensor, its design and its behavior in simulations. In Section III, a deconvolution-based, imaging method is proposed to reconstruct the dielectric profile of the sample under test with an improved spatial resolution smaller than the size of the sensor. At last, Section IV presents the performed measurements and the obtained results of capabilities of several designed sensors, and the validation of the reconstruction method.

\section{REQUIREMENTS AND DESIGN OF THE COMPLEMENTARY SPLIT-RING RESONATOR}

Since the exact sensed area should be well-defined, the outside of the balloon, i.e. the side closest to the tissue, is completely metallized and acts as ground plane. It will shield the EM waves, which are travelling towards and away from the sensor, from any interaction with other sample areas that are currently not under investigation and that could influence the measurement. To perform the measurement at the desired area, the ground plane will be interrupted and opened, and therefore a defected ground plane sensor is our preferred option. Additionally, the sensing topology should have a small footprint to increase the spatial resolution. Furthermore, a resonant sensor will achieve the highest sensitivity [26] and in order to improve the quality factor ( $\mathrm{Q}$ factor) of this resonance, a lower resonant frequency is preferred since dielectric losses increase with increasing frequencies regarding our MUT. While considering all aforementioned requirements, a complementary split-ring resonator (CSRR) is a defected ground plane sensor while offering a low resonant frequency relative to its size, and is therefore selected in this work.

The CSRR itself has already been studied thoroughly, its equivalent electrical circuit is well-known [28], and it has already been employed for liquid characterization [29] as well

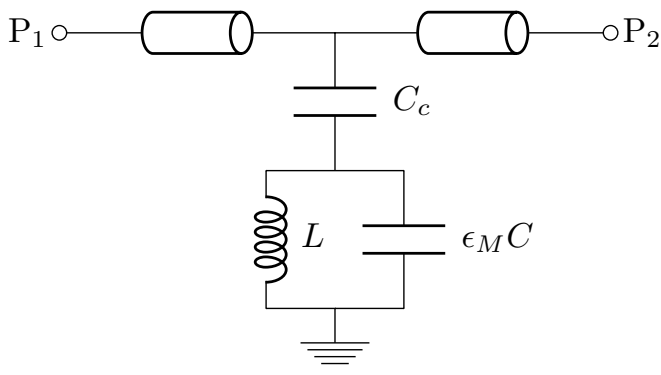

(b)

Fig. 1: (a) Schematic representation of the CSRR (transparent gold) with width $W$, spacing $S$, trace width $T$, and gap $G$, as well as the underlying microstrip transmission line on the bottom of the substrate (brown). (b) The equivalent electrical schematic of the CSRR where the transmission line is coupled to the rings through capacitor $C_{c}$. The behavior of the rings is represented by the parallel circuit of an inductor $L$ and the capacitor $C$ which depends on the permittivity $\epsilon_{M}$ of the MUT. 
as theranostic therapies [30]. Additionally, the sensing toplogy easily allows parallelization of multiple sensors coupled to a single transmission line [31]. In Fig. 1a, a schematic representation of the CSRR is shown and its design parameters are identified. CSRR's exist in circular, oval and square shapes. Here, a square-shaped design is chosen since it minimizes the resonant frequency given the available footprint [32]. Secondly, we opted for exclusive electrical excititation of the CSRR without cross-polarization by positioning the gaps orthogonally to the microstrip line [33]. Therefore, only a coupling capacitor $C_{c}$ is present in the equivalent circuit in Fig. $1 \mathrm{~b}$ between the transmission line and the rings. The behavior of the rings is represented by the parallel circuit

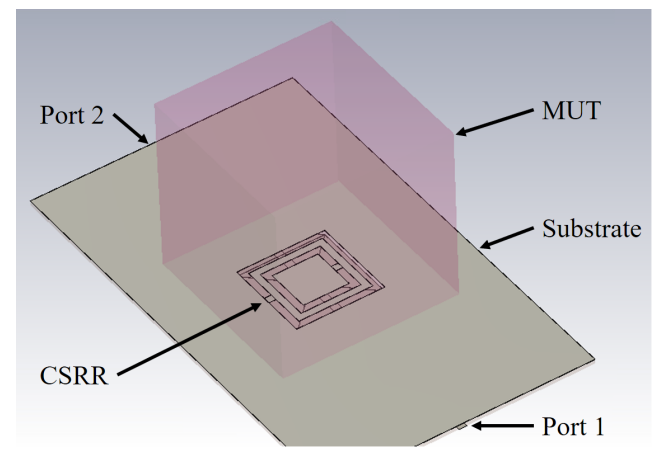

(a) An annotated illustration of the simulation setup used in CST. (The tape in between the MUT and the substrate, as well as the PMMA enclosure around the MUT are omitted for clarity.)

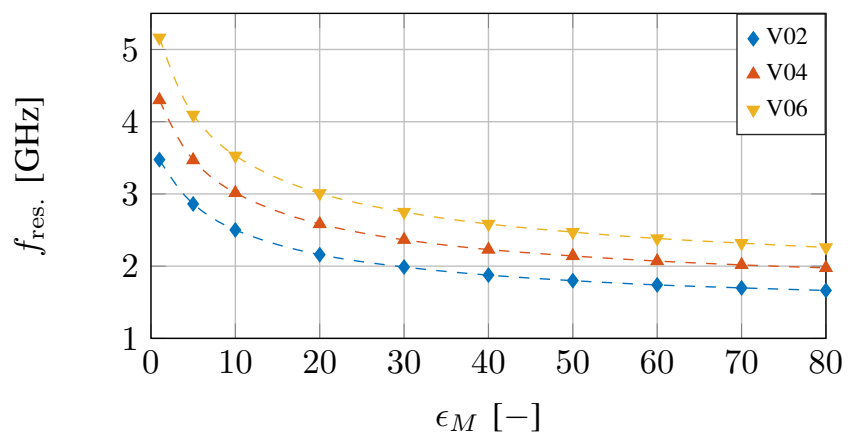

(c) The resonant frequency of the three different designs as a function of an MUT with a variable $\epsilon_{M}$ and no dielectric losses.

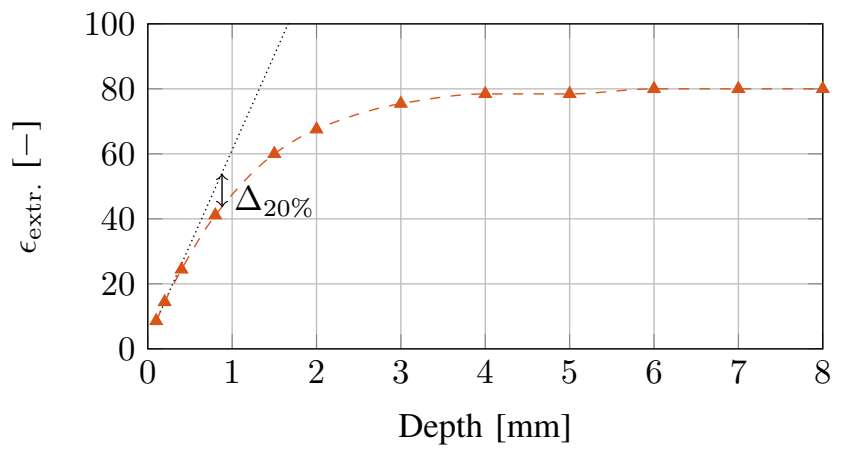

(e) Calculation of the effective penetration depth [27] of design V04 by comparing the extracted effective $\epsilon_{\text {extr. }}(\boldsymbol{\Delta})$ to its expected linear behavior ( .......) and determining the $\Delta_{20 \%}$ difference. of an inductor $L$ and the capacitor $C$ which depends on the permittivity $\epsilon_{M}=\epsilon^{\prime}-j \epsilon^{\prime \prime}$ of the MUT.

When evaluating the equivalent circuit of the CSRR, one can observe that a series resonance shorts the transmission line, which introduces a transmission zero in the structure at a MUT-dependent resonant frequency:

$$
f_{\text {res. }}=\frac{1}{2 \pi \sqrt{L \times\left(C_{c}+\epsilon_{M} C\right)}} .
$$

The values of $L$ and $C$ are directly determined by the physical dimensions of the CSRR. Increasing the circumference of the ring will result in a larger equivalent inductance $L$ whereas reducing the spacing between the rings will lead

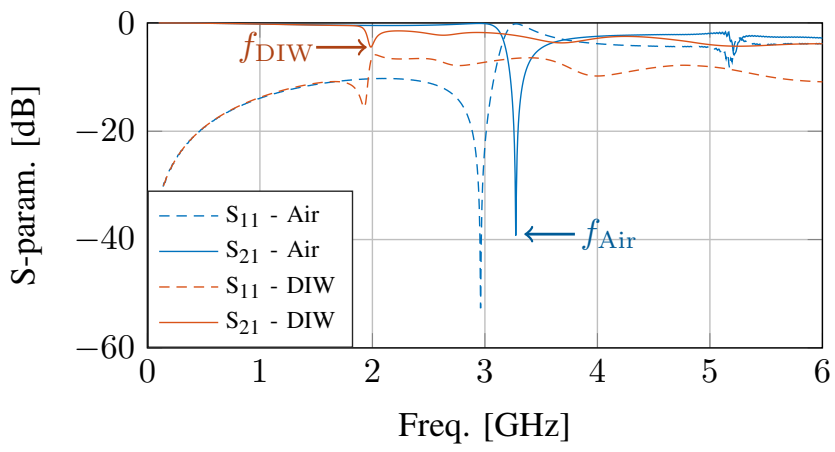

(b) The simulated reflection $\left(\mathrm{S}_{11}\right)$ and transmission $\left(\mathrm{S}_{21}\right)$ parameters of design V04 loaded with air and DIW. (Arrows indicate the corresponding resonant frequencies.)

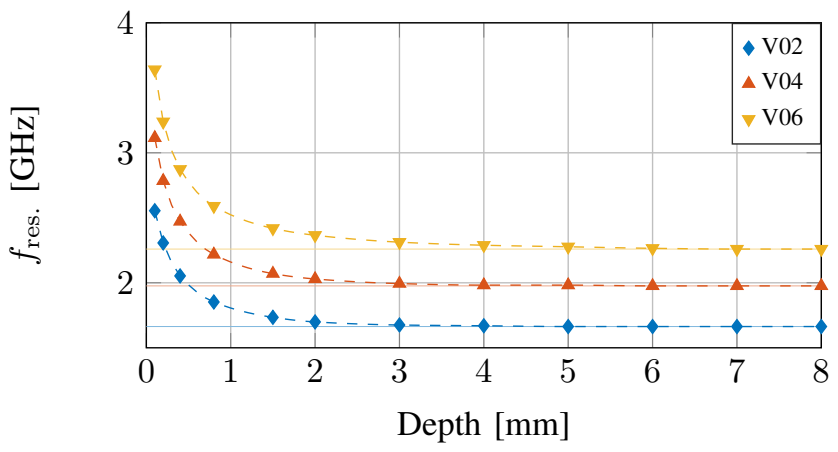

(d) The resonant frequency of the three different designs as a function of the height of the MUT $\left(\epsilon_{M}=80\right.$ and $\left.\tan \delta=0\right)$.

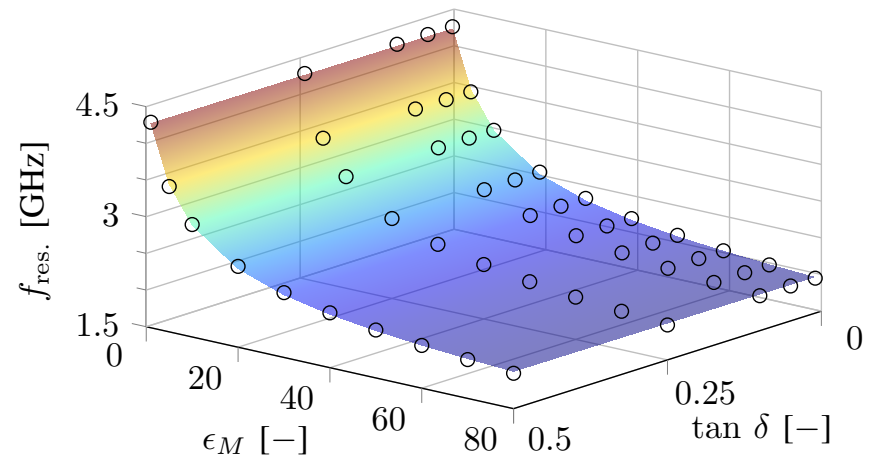

(f) Discrete points representing the resonant frequency of the V04 design as a function of the dielectric parameters of the MUT $\left(\epsilon_{M}\right.$ and $\tan \delta)$, combined with the surface fit from (2).

Fig. 2: An overview of the simulation setup in CST and the obtained simulation results for the three different designs. 
to an increased equivalent capacitance $C$. However, reducing the spacing between the rings results in a more confined electric field that limits the effective penetration depth in the tissue. In the interest of analyzing this trade-off between sensitivity and effective penetration depth, we simulated and manufactured three different devices. To maintain symmetry in the design, we opted for equal dimensions regarding the spacing $S$, the trace width $T$, and the gap $G$ (as defined in Fig. 1a). Throughout the different designs, these dimensions varied between $0.2 \mathrm{~mm}, 0.4 \mathrm{~mm}$, and $0.6 \mathrm{~mm}$ and from now on we will refer to them as design V02, V04, and V06 respectively. Since we are aiming to design a relatively small sensor that can be embedded on an inflatable balloon, we therefore limited the width $W$ to $5 \mathrm{~mm}$.

The three different designs were simulated in CST Studio Suite 2018. A schematic of the used simulation setup is shown in Fig. 2a with a $0.25 \mathrm{~mm}$ thick Rogers RO4350B substrate. To ensure a fixed MUT volume, a polymethylmethacrylate (PMMA) enclosure surrounds the MUT around the sides and a thin $100 \mu \mathrm{m}$ plastic tape seals the the bottom side of the enclosure. In Fig. 2b, the scattering parameters (S-parameters) of the CST simulations are presented for the V04 design loaded with air and with $20^{\circ} \mathrm{C}$ distilled water (DIW) [34]. To evaluate the sensitivity of the design, we ran several simulations while varying the permittivity of the MUT, $\epsilon_{M}$, for the three designs. The results from these simulations are shown in Fig 2c where the resonant frequency is displayed with respect to $\epsilon_{M}$. These simulation results confirm that the highest sensitivity can be observed for design V06, i.e. the design with the largest spacing.

Apart from the sensitivity, simulations were also used to predict the effective penetration depth of the different sensors. Here, the height of the MUT was varied as suggested in [27]. Thus a two-layered mixture of MUT and air was sensed and the effective permittivity value was reflected in the resonant frequency. To ensure that the maximum sensing depth was calculated, an MUT with a high contrast $\left(\epsilon_{M}=80\right)$ was chosen [35]. An overview of the results from these simulations can be found in Fig. 2d. The maximum depth, i.e. the depth where the device stops sensing the second layer, is determined as the point that deviates from the steady-state situation represented by the solid, constant line in Fig. 2d. Here we can observe that this depth is equal to $4.9 \mathrm{~mm}, 5.9 \mathrm{~mm}$, and $6.9 \mathrm{~mm}$ respectively for the $\mathrm{V} 02$, V04, and V06 design when employing a shape preserving interpolation (PCHIP). The V06 design, i.e. the design with the largest gaps resulted in the deepest penetration as expected. Additionally, a second method to establish the effective penetration depth was used [27]. We applied the conformal mapping approach to extract the effective permittivity of these two-layered mixtures. Since the permittivity in a two layer problem is expected to vary linearly, the second effective penetration depth was established at the point where the effective permittivity dropped $20 \%\left(\Delta_{20 \%}\right)$ below the linear relation. This second method is illustrated for the V04 design in Fig. 2e and it yielded a penetration depth of respectively $0.97 \mathrm{~mm}, 0.88 \mathrm{~mm}$, and $0.74 \mathrm{~mm}$ for the three cases. These results oppose the aforementioned findings but can be explained due to the higher linear, fitted slope of the extracted effective permittivity at small depths.

Lastly, it has been reported that dielectric losses of the MUT can have an impact on the resonant frequency of resonant sensors [36], [37]. Therefore we evaluated the resonant frequency of our V04 design for several MUT's with different $\epsilon_{M}$ and $\tan \delta$ values. The results are shown in Fig. $2 \mathrm{f}$ as discrete points. A surface as a function of $\epsilon_{M}$ and $\tan \delta$ was fitted through these discrete points and a good fit $\left(R^{2}=0.9992\right)$ was obtained:

$$
f_{\text {res. }}=1.94+\frac{23.27}{8.13+\epsilon_{M}}-\frac{1.49}{7.19+\tan \delta}[\mathrm{GHz}] .
$$

Since the influence of $\tan \delta$ in (2) is minimal, we therefore believe that we can neglect the influence of $\tan \delta$ on the resonant frequency of our sensors. When eliminating $\tan \delta$ from (2) and refitting the surface, an identical goodness of fit was achieved $\left(R^{2}=0.9992\right)$. Nevertheless, an increased $\tan \delta$ does result in a lower quality factor of the resonant frequency. This lower quality factor complicates finding $f_{\text {res. }}$ which could results in faulty readout of $f_{\text {res. }}$. These effects especially target the designs with a larger gap, i.e. the ones with a smaller $C$.

\section{DECONVOLUTION-BASED METHOD FOR IMPROVED SPATIAL RESOLUTION}

The open-ended coaxial probe (OECP) is one of the most used sensors in the collection of dielectric data but its sensing radius, which determines its spatial resolution, is rather large in the selected frequency range and is approximately equal to $\sqrt{2}$ times the aperture radius [38]. Furthermore, its elongated form factor complicates its use in planar application and therefore planar probes with broadside apertures (PPBA) were designed [39]. These PPBA demonstrated smaller fringing fields than the OECP and thus resulted in a better spatial resolution. Nevertheless, the wide-band behavior of the OECP and the PPBA makes them less accurate than resonant sensors such as a resonant patch sensor [40]. To further improve the spatial resolution of resonant sensors, sensors with a low resonant frequency relative to its size were designed [30]. However, measurements at higher frequencies will face higher dielectric losses and lead to a decreased Q-factor. Decreasing the size complicates fabrication and will limit penetration depth. These limitations drive the search for alternative solutions.

Recent developments, with techniques originating in the field of optics and radiology [43], have demonstrated the possibility to obtain spatial data of a dielectric measurement with a resolution smaller than the size of the sensor [40].

TABLE I: Comparison of the proposed sensor and algorithm in this work (T.W.) with state-of-the-art sensors and algorithms.

\begin{tabular}{|lc|c|c|c|c|}
\hline Design & & $\begin{array}{c}\text { Freq. } \\
{[\mathrm{GHz}]}\end{array}$ & $\begin{array}{c}\text { Area } \\
{\left[\mathrm{mm}^{2}\right]}\end{array}$ & $\begin{array}{c}\text { Spat. res. } \\
{[\mathrm{mm}]}\end{array}$ & $\begin{array}{c}\text { FOM }(=\lambda / \mathrm{sR}) \\
{[-]}\end{array}$ \\
\hline \hline OECP & {$[38]$} & $0.5-50$ & 3.80 & 3.1 & $194-1.9$ \\
\hline PPBA & {$[39]$} & $0.5-40$ & 3.80 & 2.2 & $272-3.4$ \\
\hline CSRR & {$[30]$} & $8-12$ & 4.80 & 4.8 & $7.8-5.2$ \\
\hline Patch & {$[40]$} & $10.5-12$ & 100 & 5.0 & $5.7-5.0$ \\
\hline SPDR & {$[41]$} & 5 & 900 & 1.0 & 60 \\
\hline SPDR & {$[42]$} & 10 & 256 & 1.0 & 30 \\
\hline \hline T.W. & & $3.5-4$ & 250 & 1.0 & $85.7-75$ \\
\hline
\end{tabular}




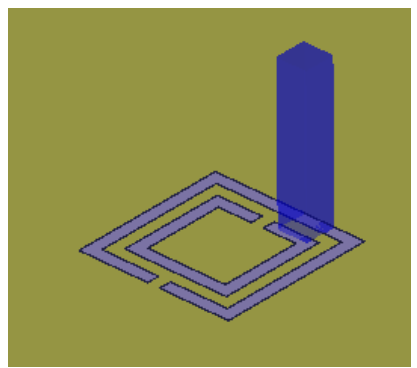

(a)

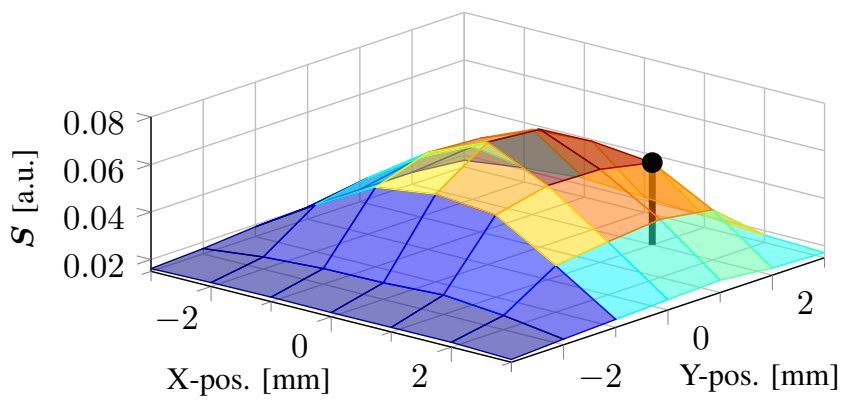

(b)

Fig. 3: (a) A 1 by 1 by $6 \mathrm{~mm}^{3}$ DIW sample positioned on the V04 design to extract the PSF of the sensor. (b) The extracted PSF $S$ of the V04 design where the black dot indicates the value corresponding to the position of the sample in (a).

The basic reconstruction principle relies on the perturbation theorem and this work makes two simplifying assumptions. Firstly, the sample is thick enough to ensure that the permittivity distribution is purely two-dimensional: $\epsilon(x, y)$. Secondly, the dielectric properties of the sample do not disturb the field pattern of the sensor and we can thus rely on the initial point spread function (PSF) measurements [41]. When scanning a sample with a known dielectric distribution in 2D, the spatial sensitivity of the sensor, i.e. the PSF of the sensor, can be extracted. Every measurement of an unknown dielectric sample thus can be considered as the sum of all corresponding PSF's over the complete distribution, i.e. a convolution of the dielectric distribution of the sample with the PSF of the sensor [44]. A deconvolution-based method, in combination with the determined PSF, can thus reconstruct the original dielectric distribution from the measured data to obtain the most-likely original image [45]. Hereby, a spatial resolution smaller than the size of the sensor can be obtained.

This technique has already been applied to split-post dielectric resonators (SPDR) with a pre-simulated electric field pattern, and it demonstrated the reconstruction of a $1 \mathrm{~mm}$ sample [41], [42]. An overview of the aforementioned sensors and the corresponding spatial resolution can be found in Table I. There, a figure-of-merit (FOM) is introduced as well to make a fair comparison between sensors operating in different frequency bands.

To extract the PSF of the V04 design from section II, a 1 by 1 by $6 \mathrm{~mm}^{3}$ DIW reference sample was enclosed in a 15 by 15 by $6 \mathrm{~mm}^{3}$ PMMA enclosure. PMMA guarantees homogeneous material properties, it has low dielectric losses, and it has the ability to obtain the desired shapes with lasercutting techniques at mm-scale. The aforementioned properties also made PMMA ideal as a background material in the different measured scenarios. Nevertheless, it is not required that the material that is used in the PSF measurements, is used in the final measurements as well: a PSF, obtained from measurements with a PMMA-background, allows dielectric imaging of an object with a different background material as well, as long as the permittivity of this other background material is higher than the one of PMMA.

Next, this reference PMMA block was moved over a 7 by $72 \mathrm{D}$ grid with $1 \mathrm{~mm}$ spacing and, at each position, the resonant frequency was measured. The origin $(0,0)$ of the grid is located in the center of the sensor and in Fig. 3a an example of the DIW sample at position $(1,2)$ is given where the PMMA enclosure is omitted for clarity. These $f_{\text {res. }}$ are gathered in a matrix $\boldsymbol{F}_{\mathbf{R}}$. Next, $\boldsymbol{F}_{\mathbf{R}}$ is transformed to its corresponding permittivity values using a conformal mapping function $c(f)$ and the permittivity of the background material during calibration $\left(\epsilon_{\text {bg.cal. }}\right)$ is deducted:

$$
\boldsymbol{E}_{\mathbf{R}}=c\left(\boldsymbol{F}_{\mathbf{R}}\right)-\epsilon_{\text {bg.cal. }} .
$$

At last, $\boldsymbol{E}_{\mathrm{R}}$ is normalized with regards to the permittivity of the DIW sample [34] at the corresponding $f_{\text {res. }}$ to obtain $\boldsymbol{S}$, the PSF of the sensor:

$$
\boldsymbol{S}=\frac{\boldsymbol{E}_{\mathbf{R}}}{\boldsymbol{\epsilon}_{\mathbf{M U T}}\left(f_{\text {res. }}\right)-\epsilon_{\text {bg.cal. }}} .
$$

$\boldsymbol{S}$, the extracted PSF from measurements, is shown in Fig. $3 b$ and the value of $\boldsymbol{S}$ at the corresponding position of the DIW sample in Fig. 3a is illustrated with a black dot.

In order to reconstruct an unknown dielectric image $\boldsymbol{I}$, the image is 2D scanned as well on a grid with a $1 \mathrm{~mm}$ spacing. Once again, the $f_{\text {res. }}$ is measured at every position and all of them are gathered in a matrix $\boldsymbol{F}$. Next, the same conformal mapping function $c(f)$ is applied and $\epsilon_{\text {bg.cal. }}$ is deducted to obtain the convolved permittivity image $\boldsymbol{E}$ :

$$
\boldsymbol{E}=c(\boldsymbol{F})-\epsilon_{\text {bg.cal. }} .
$$

Since the permittivity is a positive value, we enforce a constraint that replaces all negative values in $\boldsymbol{E}$ with 0 . Next, we apply the Lucy-Richardson deconvolution algorithm in Matlab [46] on the data $\boldsymbol{E}$ with the extracted PSF $\boldsymbol{S}$. At last, the $\epsilon_{\text {bg.cal }}$ is added to the deconvolved result to obtain the reconstructed image $\boldsymbol{R}$ :

$$
\left.\boldsymbol{R}=(\boldsymbol{E} \circledast)^{-1} \boldsymbol{S}\right)+\epsilon_{\text {bg.cal. }} .
$$

\section{EXPERIMENTAL RESULTS AND ANALYSIS}

\section{A. Measurement setup}

The CSRR designs were fabricated with standard procedures at a commercial company on a $0.25 \mathrm{~mm}$ Rogers R4350B substrate. This substrate was chosen due to its thickness and its dielectric properties that are close to the ones of polymers in inflatable balloons. Two subminiature version A (SMA) connectors (Cinch Connectivity 142-0761-841) were soldered 


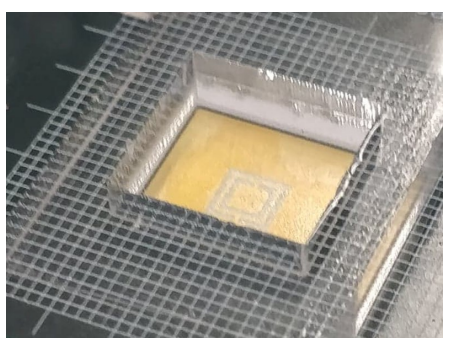

(a) An etched PMMA reservoir to hold the liquid under test during sensitivity experiments.

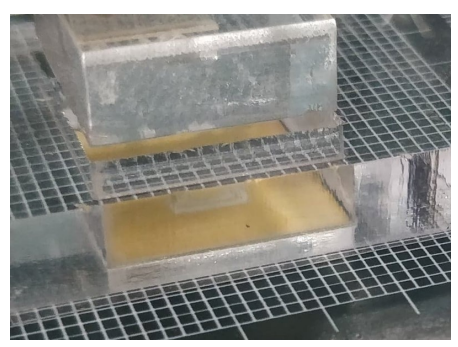

(b) A lateral moving PMMA block to establish the penetration depth of the CSRR.

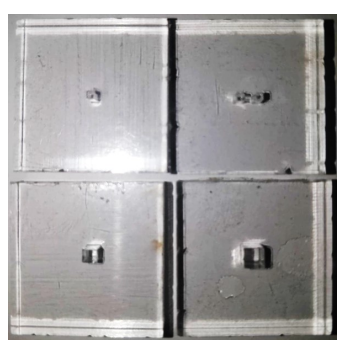

(c) The PMMA test blocks for recovering a dielectric image.

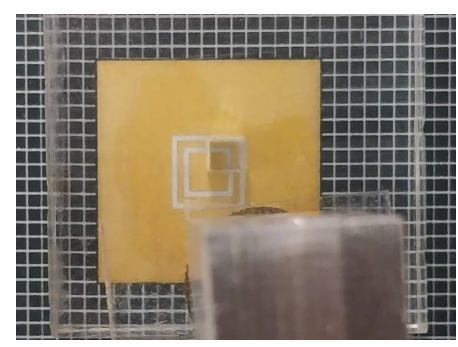

(d) A 2 by $2 \mathrm{~mm}^{2}$ PMMA test block that is positioned on the 2D grid to measure its $f_{\text {res. }}$.

Fig. 4: An overview of the measurement setups used throughout all experiments.

by hand on both sides of the transmission line. Due to thin substrate, the printed circuit board (PCB) was suspended in a PMMA setup to ensure stability. The different measurement setups for all conducted experiments are shown in Fig. 4. Each setup will be described in the following subsections.

S-parameters were recorded with a Keysight PNA (E8361) that was connected to the SMA connectors using two Gore Phaseflex 3GW40 cables. A system calibration with a standard short-open-load-through (SOLT) calibration kit placed the reference plane at the SMA-connectors. The intermediate frequency bandwidth (IFBW) was set to $100 \mathrm{~Hz}$ and an output power level of $-10 \mathrm{dBm}$ was applied. The PNA frequency range was set from $500 \mathrm{MHz}$ to $6 \mathrm{GHz}$ with a frequency step of $2 \mathrm{MHz}$. Due to internal reflections on the PCB, due to connector assembly, an undesired ripple was observed in the S-parameters. Therefore, an extra $6 \mathrm{~dB}$ attenuator (Agilent 8493C) was added between port 1 of the PNA and the SMA connector of the sensor to dampen the effect of these interfering reflections on the measurements.

\section{B. Analysis of sensor sensitivity}

To analyze the relation between the permittivity $\epsilon_{M}$ and the resonant frequency $f_{\text {res. }}$, a reservoir was etched into PMMA and sealed with tape at the bottom. This reservoir was filled with different liquids with different $\epsilon_{M}$ values (Fig. 4a). The same tape was used consistently throughout all other conducted experiments to produce comparable results. The effect of the tape on $f_{\text {res. }}$ is comparable to that of an air gap which can be compensated for [32].

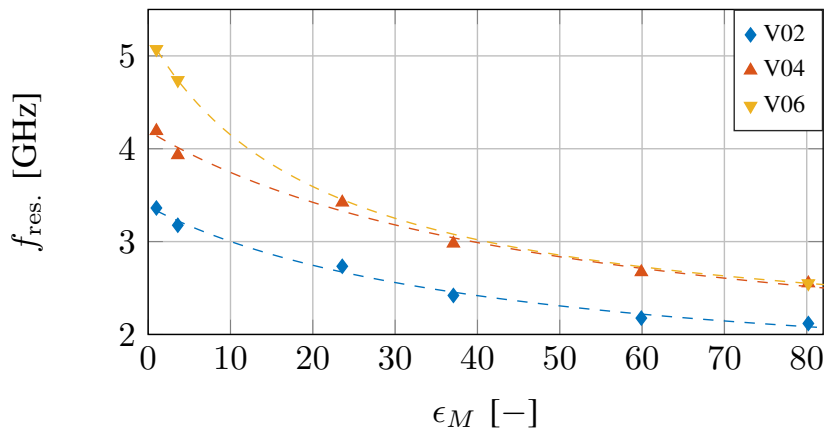

Fig. 5: The resonant frequency of the three different designs as a function of an MUT with a variable $\epsilon_{M}$.
Six different materials with a different $\epsilon_{M}$ were used to study the behavior of the resonant frequency $f_{\text {res. }}$ : air, PMMA, $60 \%$ IPA mixture, 30\% IPA mixture, 10\% IPA mixture, and DIW. The obtained results are displayed in Fig. 5 together with a fitted curve following the previously introduced model:

$$
f_{\text {res. }}=a+\frac{b}{c+\epsilon_{M}}[\mathrm{GHz}] .
$$

This fitted model is further used as the conformal mapping function $c(f)$ which was introduced in eq. (3). The values for the three different designs can be found in Table II.

TABLE II: The parameters of the fitted model of eq. (7) for the three designs.

\begin{tabular}{|c|c|c|c|}
\hline Design & $a$ & $b$ & $c$ \\
\hline V02 & 1.394 & 84.74 & 42.71 \\
\hline V04 & 1.436 & 142.4 & 51.67 \\
\hline V06 & 1.829 & 73.44 & 21.56 \\
\hline
\end{tabular}

Additionally, similar behavior as in simulations is observed and a shift in $f_{\text {res. }}$ of $-15.7 \mathrm{MHz},-20.7 \mathrm{MHz}$, and $-31.8 \mathrm{MHz}$ per a.u. of $\epsilon_{M}$ for designs V02, V04, and V06, respectively, is achieved over a broad $\epsilon_{M}$ range from 1 to 80 . These obtained values demonstrate that the designs with a larger gap are more sensitive towards changes in $\epsilon_{M}$. Nevertheless, increasing the gap does result in a lower Q factor. Therefore, several $f_{\text {res. }}$ values could not be determined for the V06 design and are missing in Fig. 5. This could explain the overlap of the curves of V04 and V06 as well.

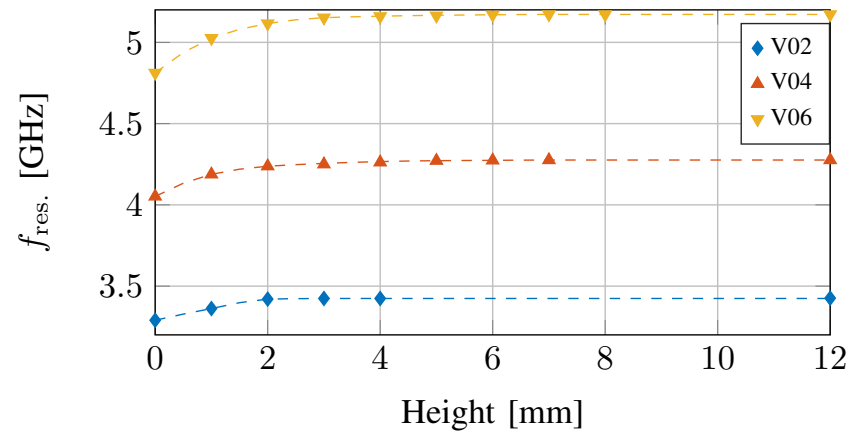

Fig. 6: The resonant frequency of the three different designs as a function of the height of the lateral moving PMMA block. 


\section{Analysis of penetration depth}

To estimate the penetration depth of the different designs, a PMMA block was mounted to a probe holder to change its lateral position with regards to the sensor (Fig. 4b). At the first measurement, the PMMA block was contacting the sensor (height $=0 \mathrm{~mm}$ ) and $f_{\text {res. }}$. was measured. Next, the PMMA block was repeatedly raised with $1 \mathrm{~mm}$ steps to perform the following measurements. The obtained results for the three designs are displayed in Fig. 6.

During these measurements, the MUT is a mixture of air and PMMA and the $f_{\text {res. }}$ of the sensor will change accordingly. When the gap between the PMMA block and the sensor exceeds the penetration depth of the sensor, the sensor only measures air and $f_{\text {res. }}$ will thus be constant. Following this line of reasoning, the maximal obtained penetration depth values are $3 \mathrm{~mm}, 6 \mathrm{~mm}$, and $7 \mathrm{~mm}$ for designs V02, V04, and V06, respectively. The obtained values are well in agreement with the results from the simulations as discussed in Section II. Therefore, we can conclude that designs with a larger gap are subject to dielectric changes at greater depth.

\section{Spatial resolution experiments and results}

In order to generate spatial dielectric distributions for the measurement of the PSF as well as several test cases, cavities are etched in PMMA test blocks (Fig. 4c). These cavities are sealed with the aforementioned tape and filled with a liquid with a specific $\epsilon_{M}$. Next, the PMMA test blocks are mounted on a probe holder that horizontally maneuvers them over a $2 \mathrm{D}$ grid with steps of $1 \mathrm{~mm}$ (Fig. 4d). In a first PMMA test block, a 1 by $1 \mathrm{~mm}^{2}$ gap is etched and filled with DIW to extract the PSF of the V04 design as explained in Section III.

The spatial resolution of a system is determined by its ability to separate two closely spaced, identical peaks. According to the Rayleigh criterion, the peaks can be resolved if the peakto-valley ratio is smaller than 0.80 [40], i.e. if the value of the valley lies lower than the reference value:

$$
\epsilon_{\text {ref. }}=\left(\frac{\epsilon_{\mathrm{M}(\text { Peak 1) }}+\epsilon_{\mathrm{M}(\text { Peak 2) }}}{2}\right) \times 0.8 \text {. }
$$

Theretofore, a second test block was fabricated with two 1 by $1 \mathrm{~mm}^{2}$ cavities, filled with DIW, with a $2 \mathrm{~mm}$ gap in

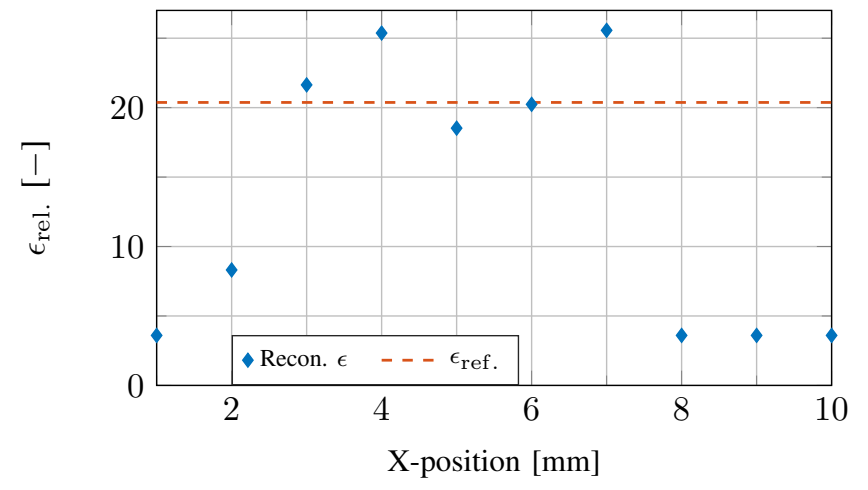

Fig. 8: The reconstructed permittivity distribution of two closely spaced, identical DIW peaks together with the Rayleigh reference value $\epsilon_{\text {ref. }}$ (eq. (8)).

between them. Fig. 7 shows its dielectric distribution $I$, its measured dielectric properties $\boldsymbol{E}$, and its dielectric properties $\boldsymbol{R}$. The permittivity values at the center line were extracted from Fig. 7 and are displayed in Fig. 8. Thus, we can conclude that the spatial resolution of our V04 design is equal to $2 \mathrm{~mm}$ which is clearly smaller than the size of the sensor.

At last, five additional different scenarios where fabricated, measured, and their dielectric distribution was reconstructed: a 3 by $3 \mathrm{~mm}^{2}$ block of DIW, a 2 by $2 \mathrm{~mm}^{2}$ block of DIW, a 2 by $2 \mathrm{~mm}^{2}$ block of $30 \%$ IPA mixture, a 2 by $2 \mathrm{~mm}^{2}$ block of DIW partially surrounded by DIW, and two 3 by $1 \mathrm{~mm}^{2}$ blocks of DIW. All of these additional results are displayed in Fig. 9 together with an outline of the original object. The results demonstrate that objects smaller than the sensor can be detected and reconstructed and that different permittivity values can be distuinguished as well.

At last, the Signal-to-Clutter ratio (SCR) [47] characterizes how well these objects $\epsilon_{\mathrm{obj}}$. can be detected with respect to the clutter in the background $\left(\epsilon_{\mathrm{cl} . b g} .-\epsilon_{\mathrm{bg}}\right)$ by comparing the maximum reconstructed value outside the object to the maximum reconstructed value within the object:

$$
\mathrm{SCR}=\frac{\max \left(\epsilon_{\text {cl.bg. }}-\epsilon_{\text {bg. }}\right)}{\max \left(\epsilon_{\text {obj. }}\right)} .
$$

The SCR values in the last presented five scenarios are

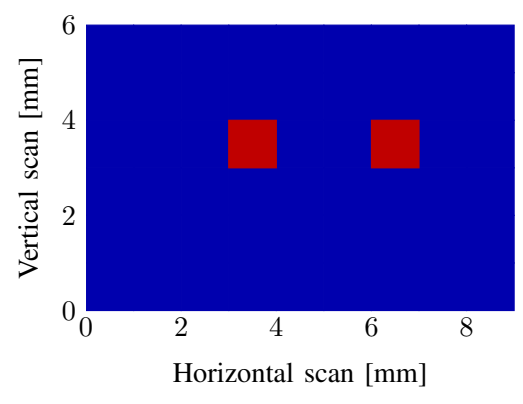

(a)

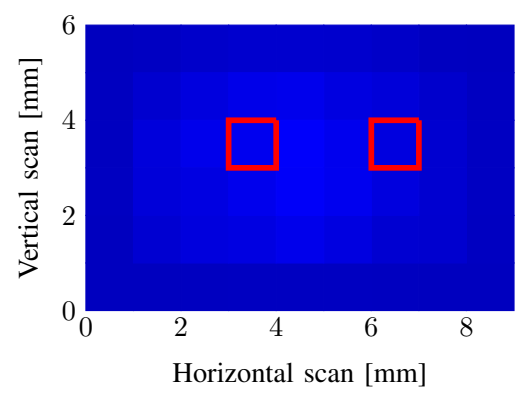

(b)

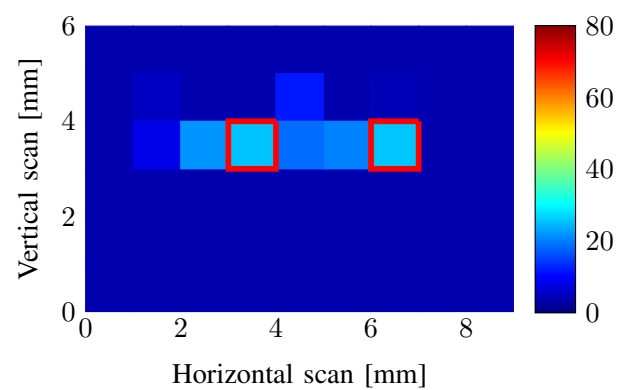

(c)

Fig. 7: The dielectric reconstruction of two peaks of DIW ( $\epsilon_{\mathrm{DIW}} \approx 75$ ). (a) represents the dielectric properties of the target $(\boldsymbol{I})$. (b) and (c) respectively represent the dielectric properties of the measured $(\boldsymbol{E})$ and reconstructed dielectric properties $(\boldsymbol{R})$, and the red lines indicate the circumference of the original object. 


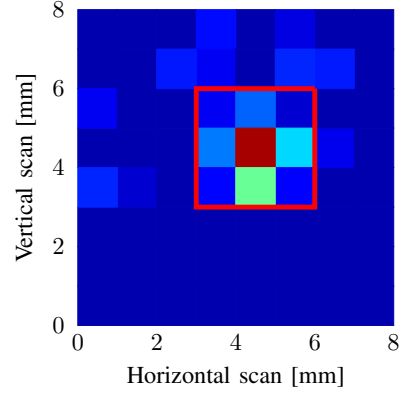

(a) 3 by $3 \mathrm{~mm}^{2}$ block of DIW $\left(\epsilon_{\mathrm{DIW}} \approx 75\right)$.
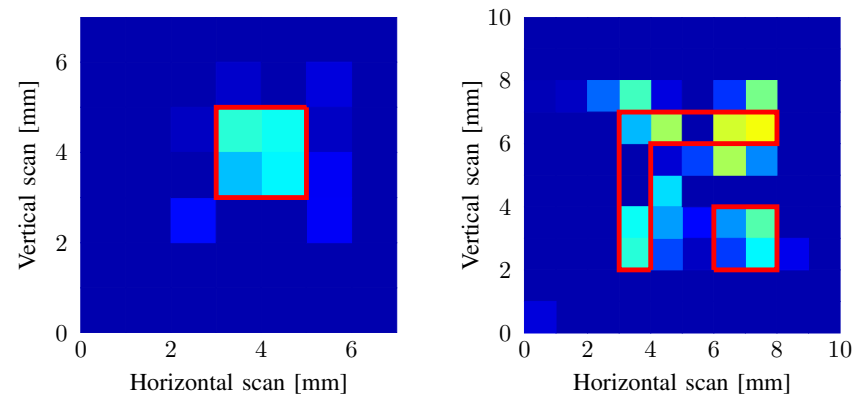

(c) 2 by $2 \mathrm{~mm}^{2}$ block of $30 \%$ IPA (d) 2 by $2 \mathrm{~mm}^{2}$ block of DIW, mixture $\left(\epsilon_{30 \% \text { IPA }} \approx 37\right)$.

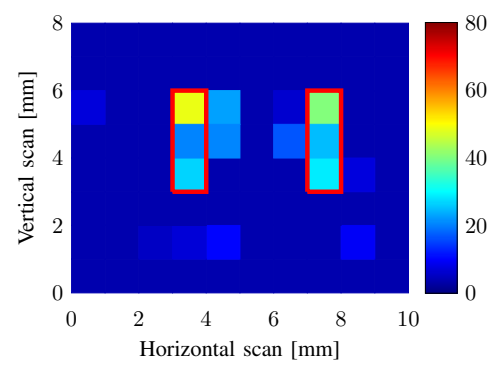

(e) Two times a 3 by $1 \mathrm{~mm}^{2}$ block of DIW.

Fig. 9: The dielectric reconstruction of five additional scenarios using the deconvolution-based approach. The red lines indicate the circumference of the original object and the colorbar in (e) applies to the data in all five figures.

respectively equal to $12.0 \%, 21.9 \%, 21.4 \%, 66.8 \%$, and $39.4 \%$. At last, the results show that no offset is present between the position of the original and the reconstructed object.

\section{CONCLUSION AND FUTURE WORK}

In this work, we designed three 5 by $5 \mathrm{~mm}^{2}$ CSRR's, each of them with different internal dimensions. As demonstrated in the simulations and in the measurements, a higher sensitivity $\left(-31.8 \mathrm{MHz}\right.$ per a.u. of $\epsilon_{M}$ for design V06) as well as a deeper penetration into the MUT ( $7 \mathrm{~mm}$ for design V06) was achieved with the designs with larger dimensions. Nevertheless, the design with larger dimensions suffered from a decreased $\mathrm{Q}$ factor which made the readout of the resonant frequency more prone to errors.

A deconvolution-based method was proposed to improve the spatial resolution of the CSRR beyond the size of the sensor.
Therefore, the PSF of the sensor was derived from scanning a known dielectric distribution on a $2 \mathrm{D}$ grid and recording the position-dependent resonant frequencies. The dielectric distribution was reconstructed in six different scenarios. The results demonstrate that objects smaller than the sensor can be detected and reconstructed and that different permittivity values can be distinguished as well. Comparing the obtained results with the state-of-the-art (Table I), the proposed sensor was able to distinguish $1 \mathrm{~mm}$ objects at $3.5-4 \mathrm{GHz}$ which resulted in a FOM of 85.7 - 75. Applying the Rayleigh criterion to two closely located, identical peaks, a spatial resolution of $2 \mathrm{~mm}$ was determined. Additionally, a good SCR value was obtained and no offset was present.

In future work, we will continue working with the presented sensing topology and designs, and implement them on flexible substrates and polymers, e.g. Liquid Crystal Polymer (LCP). A first step towards this implementation would be executed on an inflatable balloon with non-compliant zones to limit the geometrical deformation in the area surrounding the sensors. Depending on the impact on the resonant frequency, an extra elongation-based calibration step could be considered. Additionally, multiple sensors at specific resonant frequencies will be integrated together and coupled to a single transmission line to accomplish spatial positioning through frequency multiplexing.

\section{ACKNOWLEDGMENT}

The authors would like to acknowledge Rudi Casteels at the Central Mechanical Workshop (CMW) at the Department of Electrical Engineering, Leuven, Belgium, for advice on the design of the PMMA measurement setup and for the fabrication of the PMMA test setup in this work, as well as Meng Zhang for the preparation of the used IPA mixtures.

\section{REFERENCES}

[1] F. Bray, J. Ferlay, I. Soerjomataram, R. L. Siegel, L. A. Torre, and A. Jemal, "Global cancer statistics 2018: GLOBOCAN estimates of incidence and mortality worldwide for 36 cancers in 185 countries," $C A$ : A Cancer Journal for Clinicians, vol. 68, no. 6, pp. 394-424, 2018.

[2] R. Sharma, "An examination of colorectal cancer burden by socioeconomic status: evidence from GLOBOCAN 2018," EPMA Journal, vol. 11, no. 1, pp. 95-117, 2019

[3] M. Arnold, M. S. Sierra, M. Laversanne, I. Soerjomataram, A. Jemal, and F. Bray, "Global patterns and trends in colorectal cancer incidence and mortality," Gut, vol. 66, no. 4, pp. 683-691, 2016.

[4] B. Levin, D. A. Lieberman, B. Mcfarland, K. S. Andrews, D. Brooks, J. Bond, C. Dash, F. M. Giardiello, S. Glick, D. Johnson, and et al., "Screening and surveillance for the early detection of colorectal cancer and adenomatous polyps, 2008: A joint guideline from the American cancer society, the US multi-society task force on colorectal cancer, and the American college of radiology," Gastroenterology, vol. 134, no. 5, pp. 1570-1595, 2008.

[5] S. Winawer, R. Fletcher, L. Miller, F. Godlee, M. Stolar, C. Mulrow, S. Woolf, S. Glick, T. Ganiats, J. Bond, and et al., "Colorectal cancer screening: Clinical guidelines and rationale," Gastroenterology, vol. 112, no. 2, pp. 594-642, 1997.

[6] S. Winawer, R. Fletcher, D. Rex, J. Bond, R. Burt, J. Ferrucci, T. Ganiats, T. Levin, S. Woolf, D. Johnson, L. Kirk, S. Litin, and C. Simmang, "Colorectal cancer screening and surveillance: Clinical guidelines and rationale:update based on new evidence," Gastroenterology, vol. 124, no. 2, pp. 544-560, 2003.

[7] S. B. Ahn, D. S. Han, J. H. Bae, T. J. Byun, J. P. Kim, and C. S. Eun, "The miss rate for colorectal adenoma determined by quality-adjusted, back-to-back colonoscopies," Gut and Liver, vol. 6, no. 1, pp. 64-70, 2012. 
[8] "The Paris endoscopic classification of superficial neoplastic lesions: esophagus, stomach, and colon," Gastrointestinal Endoscopy, vol. 58, no. 6, 2003.

[9] L. Xiang, Q. Zhan, X.-H. Zhao, Y.-D. Wang, Y.-Z. Xu, A.-M. Li, W. Gong, Y. Bai, F.-C. Zhi, S.-D. Liu, and et al., "Risk factors associated with missed colorectal flat adenoma: A multicenter retrospective tandem colonoscopy study," World Journal of Gastroenterology, vol. 20, no. 31, pp. 10927-10937, 2014

[10] V. Konda, S. S. Chauhan, B. K. A. Dayyeh, J. H. Hwang, S. Komanduri, M. A. Manfredi, J. T. Maple, F. M. Murad, U. D. Siddiqui, S. Banerjee, and et al., "Endoscopes and devices to improve colon polyp detection," Gastrointestinal Endoscopy, vol. 81, no. 5, pp. 1122-1129, 2015.

[11] F. Topfer and J. Oberhammer, "Millimeter-wave tissue diagnosis: The most promising fields for medical applications," IEEE Microwave Magazine, vol. 16, no. 4, pp. 97-113, 2015.

[12] K. Grenier, D. Dubuc, T. Chen, F. Artis, T. Chretiennot, M. Poupot, and J.-J. Fournie, "Recent advances in microwave-based dielectric spectroscopy at the cellular level for cancer investigations," IEEE Transactions on Microwave Theory and Techniques, vol. 61, no. 5, pp. 2023-2030, 2013.

[13] Y. Feldman, I. Ermolina, and Y. Hayashi, "Time domain dielectric spectroscopy study of biological systems," IEEE Transactions on Dielectrics and Electrical Insulation, vol. 10, no. 5, pp. 728-753, 2003.

[14] A. Martellosio, M. Pasian, M. Bozzi, L. Perregrini, A. Mazzanti, F. Svelto, P. E. Summers, G. Renne, L. Preda, and M. Bellomi, "Dielectric properties characterization from 0.5 to $50 \mathrm{GHz}$ of breast cancer tissues," IEEE Transactions on Microwave Theory and Techniques, vol. 65 , no. 3, pp. 998-1011, 2017

[15] T. M. Grzegorczyk, P. M. Meaney, P. A. Kaufman, R. M. diFlorioAlexander, and K. D. Paulsen, "Fast 3-D tomographic microwave imaging for breast cancer detection," IEEE Transactions on Medical Imaging, vol. 31, no. 8, pp. 1584-1592, 2012.

[16] X. Li and S. C. Hagness, "A confocal microwave imaging algorithm for breast cancer detection," IEEE Microwave and Wireless Components Letters, vol. 11, no. 3, pp. 130-132, 2001.

[17] W. Shao and T. Mccollough, "Advances in microwave near-field imaging: Prototypes, systems, and applications," IEEE Microwave Magazine, vol. 21, no. 5, pp. 94-119, 2020.

[18] L. Zhang, C. Bounaix Morand du Puch, C. Dalmay, A. Lacroix A. Landoulsi, J. Leroy, C. Mlin, F. Lallou, S. Battu, C. Lautrette, and et al., "Discrimination of colorectal cancer cell lines using microwave biosensors," Sensors and Actuators A: Physical, vol. 216, pp. 405-416, 2014.

[19] A. Fornes-Leal, C. Garcia-Pardo, M. Frasson, V. P. Beltrán, and N. Cardona, "Dielectric characterization of healthy and malignant colon tissues in the 0.5-18 GHz frequency band," Physics in Medicine and Biology, vol. 61, no. 20, pp. 7334-7346, 2016.

[20] Z. Li, G. Deng, Z. Li, S. X. Xin, S. Duan, M. Lan, S. Zhang, Y. Gao, J. He, S. Zhang, and et al., "A large-scale measurement of dielectric properties of normal and malignant colorectal tissues obtained from cancer surgeries at Larmor frequencies," Medical Physics, vol. 43, no. 11 , pp. 5991-5997, 2016.

[21] M. Guardiola, S. Buitrago, G. Fernández-Esparrach, J. M. O’Callaghan, J. Romeu, M. Cuatrecasas, H. Córdova, M. A. G. Ballester, and O. Camara, "Dielectric properties of colon polyps, cancer, and normal mucosa: Ex vivo measurements from 0.5 to $20 \mathrm{GHz}$," Medical Physics, vol. 45, no. 8, pp. 3768-3782, 2018.

[22] M. Guardiola, K. Djafri, M. Challal, M. A. G. Ballester, G. FernándezEsparrach, O. Camara, and J. Romeu, "Design and evaluation of an antenna applicator for a microwave colonoscopy system," IEEE Transactions on Antennas and Propagation, vol. 67, no. 8, pp. 3768-3782, 2018.

[23] D.-H. Kim, N. Lu, R. Ghaffari, Y.-S. Kim, S. P. Lee, L. Xu, J. Wu, R.-H. Kim, J. Song, Z. Liu, and et al., "Materials for multifunctional balloon catheters with capabilities in cardiac electrophysiological mapping and ablation therapy," Nature Materials, vol. 10, no. 4, pp. 316-323, 2011.

[24] J. A. Dickson and S. K. Calderwood, "Temperature range and selective sensitivity of tumors to hyperthermia: A critical review," Ann. NY Acad. Sci., vol. 335, no. 1 Thermal Chara, pp. 180-205, 1980.

[25] C. J. Simon, D. E. Dupuy, and W. W. Mayo-Smith, "Microwave ablation: Principles and applications," Radiographics, vol. 25, pp. 69-83, 2005.

[26] L. F. Chen, C. K. Ong, C. P. Neo, and V. V. Varadan, Microwave Electronics Measurement and Materials Characterization, pp. 37-141. John Wiley \& Sons, 2005.

[27] P. M. Meaney, A. P. Gregory, J. Seppala, and T. Lahtinen, "Open-ended coaxial dielectric probe effective penetration depth determination," IEEE Transactions on Microwave Theory and Techniques, pp. 915-923, 2016.
[28] J. D. Baena, J. Bonache, F. Martin, R. M. Sillero, F. Falcone, T. Lopetegi, M. A. G. Laso, J. Garcia-Garcia, I. Gil, M. F. Portillo, and et al., "Equivalent-circuit models for split-ring resonators and complementary split-ring resonators coupled to planar transmission lines," IEEE Transactions on Microwave Theory and Techniques, vol. 53, no. 4, pp. 14511461,2005

[29] L. Su, J. Mata-Contreras, P. Vélez, A. Fernández-Prieto, and F. Martín, "Analytical method to estimate the complex permittivity of oil samples," Sensors, vol. 18, no. 4, p. 984, 2018.

[30] C. Reimann, M. Puentes, M. Maasch, F. Hübner, B. Bazrafshan, T. Vogl, C. Damm, and R. Jakoby, "Planar microwave sensor for theranostic therapy of organic tissue based on oval split ring resonators," Sensors, vol. 16 , no. 9 , p. $1450,2016$.

[31] M. Abdolrazzaghi and M. Daneshmand, "Dual active resonator for dispersion coefficient measurement of asphaltene nano-particles," IEEE Sensors Journal, vol. 17, no. 22, pp. 7248-7256, 2017.

[32] M. A. H. Ansari, A. K. Jha, and M. J. Akhtar, "Design and application of the CSRR-based planar sensor for noninvasive measurement of complex permittivity," IEEE Sensors Journal, vol. 15, no. 12, pp. 7181-7189, 2015.

[33] J. Naqui, M. Duran-Sindreu, and F. Martin, "Modeling split-ring resonator (SRR) and complementary split-ring resonator (CSRR) loaded transmission lines exhibiting cross-polarization effects," IEEE Antennas and Wireless Propagation Letters, vol. 12, pp. 178-181, 2013.

[34] W. J. Ellison, "Permittivity of pure water, at standard atmospheric pressure, over the frequency range $0-25 \mathrm{THz}$ and the temperature range 0-100 ${ }^{\circ}$ C," Journal of Physical and Chemical Reference Data, vol. 36, no. 1, pp. 1-18, 2007.

[35] A. La Gioia, M. O'Halloran, and E. Porter, "Modelling the sensing radius of a coaxial probe for dielectric characterisation of biological tissues," IEEE Access, vol. 6, pp. 46516-46526, 2018.

[36] A. Ebrahimi, W. Withayachumnankul, S. Al-Sarawi, and D. Abbott, "High-sensitivity metamaterial-inspired sensor for microfluidic dielectric characterization," IEEE Sensors Journal, vol. 14, no. 5, pp. 1345-1351, 2014.

[37] G. M. Rocco, M. Bozzi, D. Schreurs, L. Perregrini, S. Marconi, G. Alaimo, and F. Auricchio, "3-D printed microfluidic sensor in SIW technology for liquids characterization," IEEE Transactions on Microwave Theory and Techniques, vol. 68, no. 3, pp. 1175-1184, 2020.

[38] P. De Langhe, L. Martens, and D. D. Zutter, "Design rules for an experimental setup using an open-ended coaxial probe based on theoretical modelling," IEEE Transactions on Instrumentation and Measurement, vol. 43, no. 6, pp. 810-817, 1994

[39] B. Kang, J.-H. Park, J. Cho, K. Kwon, S. Lim, J. Yoon, C. Cheon, Y.-K. Kim, and Y. Kwon, "Novel low-cost planar probes with broadside apertures for nondestructive dielectric measurement of biological materials at microwave frequencies," IEEE Transactions on Microwave Theory and Techniques, vol. 53, no. 1, pp. 134-143, 2005.

[40] Y. Li, N. Bowler, and D. B. Johnson, "A resonant microwave patch sensor for detection of layer thickness or permittivity variations in multilayered dielectric structures," IEEE Sensors Journal, vol. 11, no. 1, pp. 5-15, 2011.

[41] P. Korpas, "Deconvolution-based spatial resolution improvement technique for resistivity scans acquired with split-post dielectric resonator," 2018 22nd International Microwave and Radar Conference (MIKON), 2018.

[42] M. Celuch, W. Gwarek, and A. Wieckowski, "Enhanced-resolution material imaging with dielectric resonators: a new implicit space-domain technique," 2019 IEEE MTT-S International Microwave Symposium (IMS), 2019.

[43] P. Suetens, Fundamentals of medical imaging. Cambridge University Press, 2017

[44] K. Rossmann, "Point spread-function, line spread-function, and modulation transfer function," Radiology, vol. 93, no. 2, pp. 257-272, 1969.

[45] J. G. Mcnally, T. Karpova, J. Cooper, and J. A. Conchello, "Threedimensional imaging by deconvolution microscopy," Methods, vol. 19, no. 3, pp. 373-385, 1999.

[46] "Matlab image processing toolbox," Version 9.3 - R2017b. The MathWorks, Natick, MA, USA.

[47] R. C. Conceicao, M. O'Halloran, M. Glavin, and E. Jones, "Comparison of planar and circular antenna configurations for breast cancer detection using microwave imaging," Progress In Electromagnetics Research, vol. 99, pp. 1-20, 2009. 